\title{
Defining limited stage small cell lung cancer: a radiation oncologist's perspective
}

\author{
Irfan Ahmad, ${ }^{1}$ Kundan Singh Chufal, ${ }^{2}$ Sumant Gupta, ${ }^{3,4}$ Chandi Prasad Bhatt ${ }^{1}$
}

'Department of Radiation Oncology, Batra Hospital and Medical Research Centre, New Delhi, Delhi, India

${ }^{2}$ Department of Radiation Oncology, Rajiv Gandhi Cancer Institute and Research Centre, New Delhi, Delhi, India ${ }^{3}$ Department of Medical Oncology, Batra Hospital and Medical Research Centre, New Delhi, Delhi, India

${ }^{4}$ Department of Medical Oncology, Sarvodaya Hospital, Faridabad, Haryana, India

\section{Correspondence to \\ Dr Irfan Ahmad,}

irfan.a@icloud.com

Accepted 1 January 2018

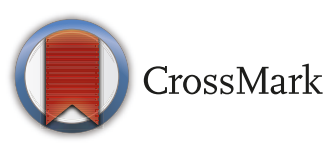

To cite: Ahmad I, Chufal KS, Gupta S, et al. BMJ Case Rep Published Online First: [please include Day Month Year]. doi:10.1136/bcr-2017223708

\section{DESCRIPTION}

Small cell lung cancer (SCLC) comprises 13\%-15\% of all diagnosed lung carcinomas and approximately $30 \%$ of those will be defined as limited stageSCLC (LS-SCLC). ${ }^{1}$ The definition of LS-SCLC has undergone many modifications and there is no universally accepted definition till date (figure 1$)^{2}$ It was first defined by the Veteran Affairs Lung cancer study Group (VALG) in 1973 and further redefined by the International Association for the Study of Lung Cancer (IASLC) and American Joint Committee on Cancer (AJCC) in 1989 and 2010, respectively. ${ }^{1}{ }^{2}$ Considerable heterogeneity exists pertaining to which definition is used for treating patients in routine practice. As a direct consequence of this, discussions often arise in multidisciplinary tumour board meetings in which different oncologists classify the same patient differently. The subsequent images demonstrate the heterogeneity in subjective classification of patients with SCLC and the role of radiotherapy (RT) in appropriate classification of SCLC.

Figure 2 demonstrates a patient with multiple pulmonary nodules in right upper lobe $\left(\mathrm{cT}_{3}\right)$ and multiple ipsilateral mediastinal nodes $\left(\mathrm{cN}_{2}\right)$, Stage IIIA. This case would be classified as LS-SCLC as per VALG and IASLC definitions and possibly extensive stage SCLC (ES-SCLC) as per AJCC definition. Treatment planning images show that if this patient were to be treated with three dimensional conformal radiotherapy (3DCRT), based on total lung minus planning target volume (TLPTV) receiving 20 Gray $(\mathrm{Gy})\left(\mathrm{V}_{20}\right)$ and spinal cord maximum dose $\left(\mathrm{D}_{\max }\right)$ parameters, proceeding with RT would be deemed intolerable and unsafe, respectively, placing this patient into ES-SCLC group. On the contrary, utilising volumetric modulated arc therapy (VMAT) planning satisfies both parameters.

Figure 3 demonstrates a patient with a pulmonary mass in left upper lobe $\left(\mathrm{cT}_{2 \mathrm{~b}}\right)$ and multiple contralateral mediastinal nodes $\left(\mathrm{cN}_{3}\right)$, Stage IIIB. This case would be classified as LS-SCLC as per IASLC and AJCC definitions and ES-SCLC as per VALG definition. Treatment planning images show that utilising 3DCRT would be intolerable as per TL-PTV $\mathrm{V}_{20}$ parameter, placing this patient in ES-SCLC group. VMAT planning adequately satisfies this parameter.

Figure 4 demonstrates a patient with a pulmonary mass in right lower lobe $\left(\mathrm{cT}_{2 \mathrm{~b}}\right)$ with multiple ipsilateral and contralateral mediastinal nodes, ipsilateral supraclavicular fossa nodes and contralateral hilar node $\left(\mathrm{cN}_{3}\right)$, Stage IIIB. This case would be classified as ES-SCLC as per VALG and IASLC

\section{Learning points}

- A thorough understanding of the differences in anatomical staging systems for small cell lung cancer $(\mathrm{SCLC})$ is essential for appropriate distinction between limited stage SCLC (LS-SCLC) and extensive stage SCLC (ES-SCLC).

- Modern radiotherapy planning techniques combined with an emphasis on objective quantification of anticipated toxicities can allow safe and tolerable treatment delivery in challenging cases of SCLC, resulting in improved treatment outcomes.

- Radiotherapy planning should be discussed in multidisciplinary tumour board meetings, prior to classifying patients as LS-SCLC or ES-SCLC, so that an appropriate treatment approach can be selected.

definitions and possibly LS-SCLC as per AJCC definition. Treatment planning images utilising VMAT demonstrate that this technique would be intolerable as per TL-PTV $V_{20}$ parameter, placing this patient in ES-SCLC group.

These cases demonstrate the following. (1) All staging systems are rarely in concordance with respect to defining LS-SCLC. (2) Subjective classification of patients based on purely anatomical definitions can lead to toxicity (disease extent underestimated) or undertreatment (disease extent overestimated). (3) Objective classification utilising modern RT planning can ensure uniformity in decision making. (4) RT planning technology can influence the decision in categorising extent of disease as LS-SCLC or ES-SCLC.

Concurrent chemoradiotherapy is the standard of care for LS-SCLC and compared with chemotherapy alone, addition of RT improves 2-year overall survival by $5 \%-7 \% .^{3}$ Therefore, emphasis should be placed on determining objectively whether RT can be delivered utilising modern RT techniques in all cases of SCLC.

Contributors IA is the treating junior consultant (radiotherapy), author of the paper, responsible for drafting the manuscript and revising it. He is the guarantor. KSC and SG are the supervising treating consultants (radiotherapy and medical oncology, respectively) and participated in article formulation, editing and oversight. CPB is the physicist, responsible for generating the radiation treatment plan, performing quality assurance of delivered plan and also participated in article editing.

Competing interests None declared.

Patient consent Obtained. 


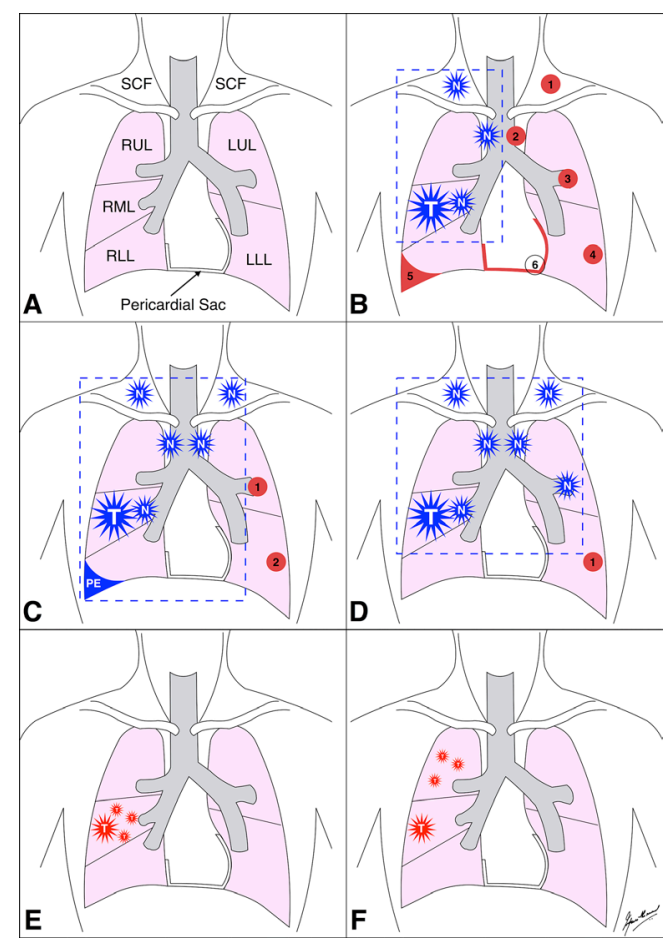

Figure 1 Diagrammatic representation of various anatomical definitions used in defining LS-SCLC. (A) Simplified line diagram depicting the areas of interest within and outside the thoracic cavity. (B) Veteran Affairs Lung cancer study Group, 1973. According to this classification system, LS-SCLC is defined as disease confined to one hemithorax (although local extension may be present) when primary tumour and regional nodes can be encompassed in a radiation port without extrathoracic metastases (except for ipsilateral SCF nodes). The anatomical regions that satisfy this criteria are shown in blue multipronged stars (T, primary tumour; N, nodes) and the encompassing radiation portal is shown in blue dashed line. The anatomical regions that are excluded are shown in red numbered circles (1, contralateral SCF nodes; 2 , contralateral hilar nodes; 3 , contralateral hilar nodes; 4 , contralateral lung nodule; 5 , malignant pleural effusion; 6 , malignant pericardial effusion). (C) International Association for the Study of Lung Cancer, 1989. According to this classification system, LS-SCLC is defined as disease confined to one hemithorax with regional lymph node involvement including ipsilateral hilar nodes, ipsilateral and contralateral mediastinal and ipsilateral and contralateral SCF nodes and should also include patients with ipsilateral pleural effusion independent whether the cytology is positive or negative. These regions are shown in blue multipronged stars (T, primary tumour; N, nodes) and the encompassing radiation portal is shown in blue dashed line. The anatomical regions that are excluded are shown in red numbered circles (1, contralateral hilar nodes; 2, contralateral lung nodule). (D-F) American Joint Committee on Cancer, 2010. According to this classification system, LS-SCLC is defined as Stages I-III (any T, any N, $M_{0}$ ) that can be safely treated with definitive radiation doses. These regions are shown in blue multipronged stars (T, primary tumour; $\mathrm{N}$, nodes) and the encompassing radiation portal is shown in blue dashed line. The anatomical regions excluded from this system is the presence of contralateral lung nodule (red numbered circle 1 ) and $T_{3}$ or $T_{4}$ primary tumour with multiple lung nodules (figure $\mathrm{E}$ and $\mathrm{F}$, respectively) that are too extensive or have tumour/nodal volume that is too large to be encompassed in a tolerable radiation plan. LLL, left lower lobe; LS-SCLC, limited stage small cell lung cancer; LUL, left upper lobe; PE, pleural effusion; RLL, right lower lobe; RML, right middle lobe; RUL, right upper lobe; SCF, supraclavicular fossa.

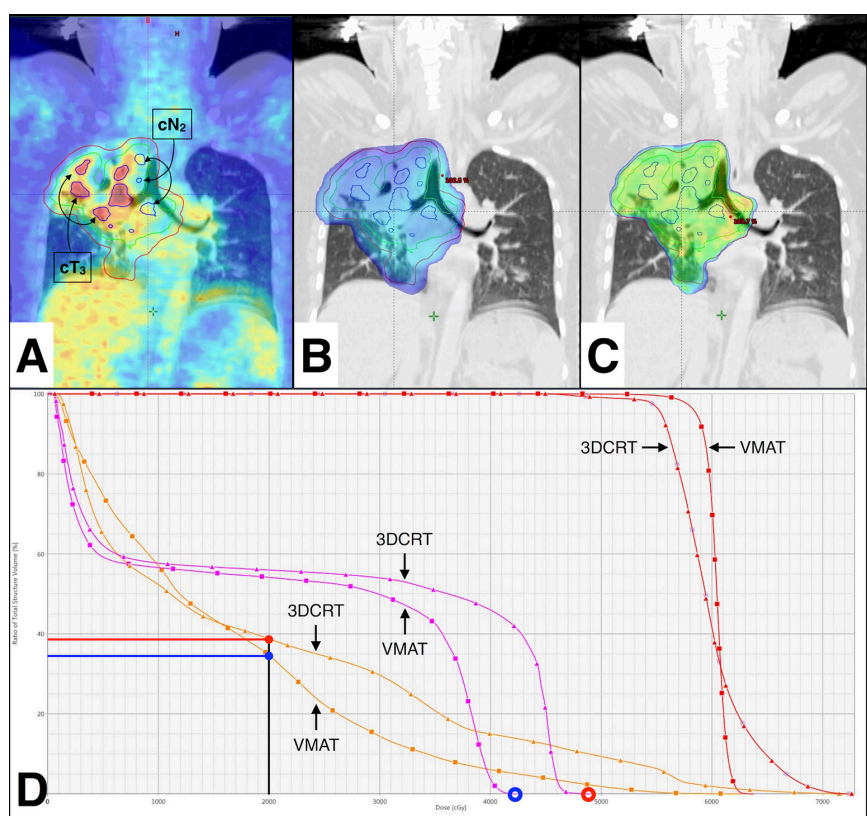

Figure 2 Staging ${ }^{18}$ flourodeoxyglucose positron emission tomography-CT ( ${ }^{18} \mathrm{FDG}$ PET-CT), and treatment planning images of a patient diagnosed with small cell lung cancer (SCLC). (A) PET-CT in coronal plane reveals multiple pulmonary nodules in right upper lobe and multiple ipsilateral mediastinal nodes (right hilar, subcarinal, right lower paratracheal and right upper paratracheal). For the purpose of treatment planning, this PET-CT was rigidly registered to simulation $\mathrm{CT}$, and gross tumour volume, clinical target volume and planning target volume (PTV) were delineated as per institutional protocol (shown in A-C as blue, green and red contours, respectively). The PTV was prescribed 60 Gray (Gy) in 30 fractions, over 6 weeks. (B) Threedimensional conformal radiotherapy (3DCRT) treatment planning images of the same patient in coronal plane, with lower limit of dose colour wash set at $95 \%$ of prescribed dose reveals that the PTV appears to be adequately covered. Dose coverage had to be compromised posteriorly, in order to spare the spinal cord as much as possible. (C) Volumetric modulated arc therapy (VMAT) treatment planning images in coronal plane with the lower limit of dose colour wash set at $95 \%$ of prescribed dose reveals that the PTV appears to be adequately covered. (D) Dose-volume histograms of 3DCRT and VMAT plans comparing total lung minus PTV (TL-PTV) (orange lines), spinal cord (magenta lines) and PTV (red lines). Comparison of the TL-PTV histograms demonstrates that the $\mathrm{V}_{20}$ metric is $34 \%$ versus $39 \%$ for VMAT and 3DCRT plans, respectively (blue and red horizontal lines, respectively). The constraint for this metric in patients treated definitively with lung carcinomas is $\mathrm{V}_{20}<35 \%$ and is associated with $<20 \%$ risk of developing radiotherapy-induced pneumonitis. ${ }^{3}$ Comparison of the PTV and spinal cord histograms demonstrates that despite efforts to protect the spinal cord in the 3DCRT plan (resulting in inferior PTV coverage compared with VMAT), the maximum dose $\left(D_{\text {max }}\right)$ of spinal cord is higher (49 Gy for 3DCRT vs 42 Gy for VMAT, blue and red circles, respectively). The constraint for this metric is $D_{\max }<45 \mathrm{~Gy} .{ }^{3}$ All other metrics were comparable between both plans. 


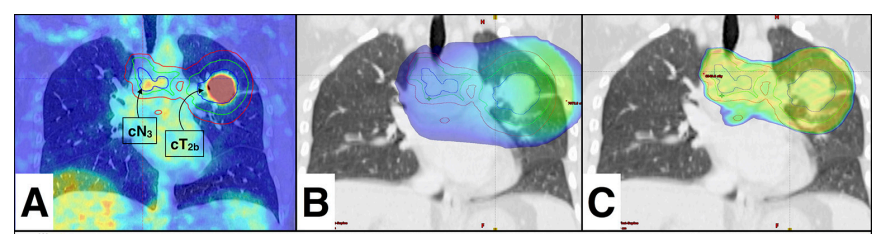

D.

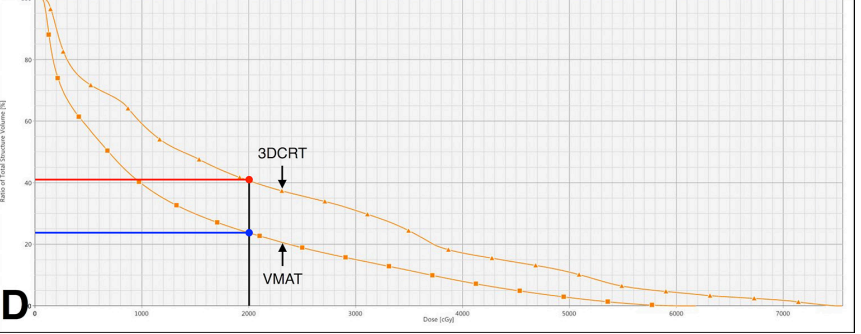

Figure 3 Staging ${ }^{18}$ flourodeoxyglucose positron emission tomography-CT $\left({ }^{18} \mathrm{FDG}\right.$ PET-CT), and treatment planning images of a patient diagnosed with SCLC. (A) PET-CT in coronal plane reveals a pulmonary mass in left upper lobe, multiple ipsilateral mediastinal nodes (left hilar, left lower paratracheal) and contralateral mediastinal nodes (right lower paratracheal). For the purpose of treatment planning, this PET-CT was rigidly registered to simulation $\mathrm{CT}$, and gross tumour volume, clinical target volume and planning target volume (PTV) were delineated as per institutional protocol (shown in $\mathrm{A}-\mathrm{C}$ as blue, green and red contours, respectively). The PTV was prescribed 60 Gray (Gy) in 30 fractions, over 6 weeks. (B) Three-dimensional conformal radiotherapy (3DCRT) treatment planning images of the same patient in coronal plane, with lower limit of dose colour wash set at $95 \%$ of prescribed dose reveals that the PTV appears to be adequately covered. (C) Volumetric modulated arc therapy (VMAT) treatment planning images in coronal plane with the lower limit of dose colour wash set at $95 \%$ reveals that the PTV appears to be adequately covered. (D) Dosevolume histogram of 3DCRT and VMAT plans comparing total lung minus PTV (orange lines) demonstrates that the $\mathrm{V}_{20}$ metric is $40 \%$ versus $22 \%$, respectively (blue and red horizontal lines, respectively). All other metrics were comparable between both plans.

Provenance and peer review Not commissioned; externally peer reviewed.

(c) BMJ Publishing Group Ltd (unless otherwise stated in the text of the article) 2018. All rights reserved. No commercial use is permitted unless otherwise expressly granted.

\section{REFERENCES}

1 Kalemkerian GP, Gadgeel SM. Modern staging of small cell lung cancer. J Nat/ Compr Canc Netw 2013;11:99-104.

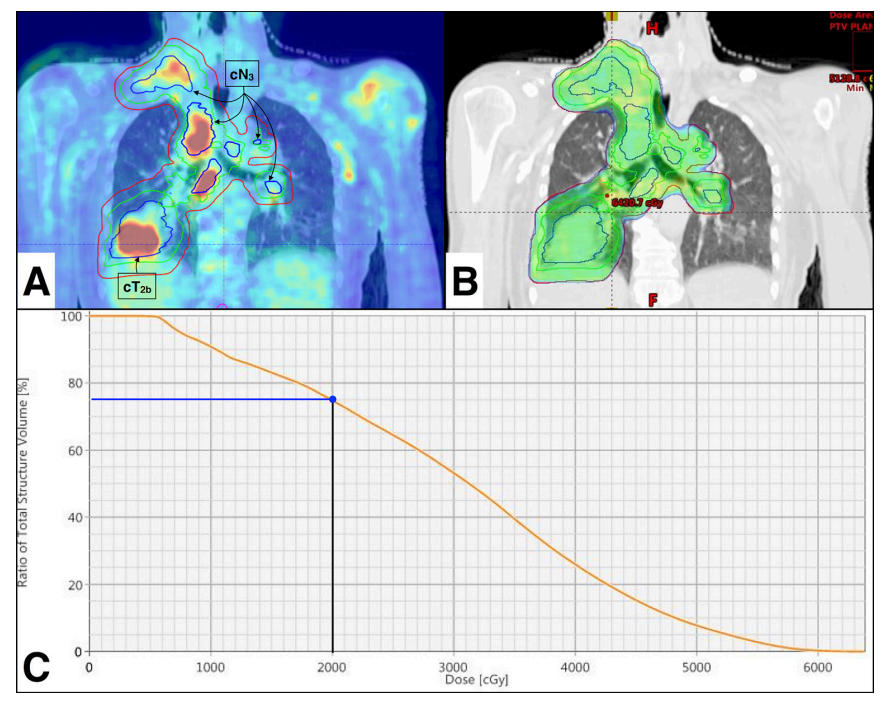

Figure 4 Staging ${ }^{18}$ flourodeoxyglucose positron emission tomography-CT $\left({ }^{18} \mathrm{FDG}\right.$ PET-CT), and treatment planning images of a patient diagnosed with small cell lung cancer (SCLC). (A) PET$\mathrm{CT}$ in coronal plane reveals a pulmonary mass in right lower lobe, multiple ipsilateral mediastinal nodes (right hilar, right lower and upper paratracheal, subcarinal), ipsilateral supraclavicular nodes and contralateral mediastinal nodes (right lower paratracheal, right hilar). For the purpose of treatment planning, this PET-CT was rigidly registered to simulation $\mathrm{CT}$, and gross tumour volume, clinical target volume and planning target volume (PTV) were delineated as per institutional protocol (shown in A and B as blue, green and red contours, respectively). The PTV was prescribed 60 Gray (Gy) in 30 fractions, over 6 weeks. (B) Volumetric modulated arc therapy (VMAT) treatment planning images in coronal plane with the lower limit of dose colour wash set at $95 \%$ of prescribed dose reveals that the PTV appears to be adequately covered. (C) Dose-volume histogram of VMAT plan showing total lung minus PTV (orange line). The $\mathrm{V}_{20}$ metric is $75 \%$ (blue horizontal line).

2 National Cancer Institute. Small Cell Lung Cancer Treatment (PDQ®)-Health Professional Version. 2017 https://www.cancer.gov/types/lung/hp/small-cell-lungtreatment-pdq\#section/_37 (accessed 19 Nov 2017).

3 Rengan R, Chetty IJ, Decker R, et al. Lung cancer. In: Halperin EC, Brady LW, Wazer DE, Perez CA. eds. Perez \& Brady's principles and practice of radiation oncology. 6th ed. Philadelphia: Lippincott Williams \& Wilkins, 2013:938-73.

Copyright 2017 BMJ Publishing Group. All rights reserved. For permission to reuse any of this content visit

http://group.bmj.com/group/rights-licensing/permissions.

BMJ Case Report Fellows may re-use this article for personal use and teaching without any further permission.

Become a Fellow of BMJ Case Reports today and you can:

- Submit as many cases as you like

- Enjoy fast sympathetic peer review and rapid publication of accepted articles

- Access all the published articles

- Re-use any of the published material for personal use and teaching without further permission

For information on Institutional Fellowships contact consortiasales@bmjgroup.com

Visit casereports.bmj.com for more articles like this and to become a Fellow 